\title{
CXCL13 CSF level inversely correlates with duration of disease in primary progressive multiple sclerosis
}

\author{
Jacek Losy' ${ }^{1,2}$, Piotr Iwanowski ${ }^{1,2}$, Elżbieta Kaufman', Marlena Wójcicka \\ ${ }^{1}$ Department of Clinical Neuroimmunology, Chair of Neurology, Poznan University of Medical Sciences, Poland \\ 2 Department of Neurology, Chair of Neurology, Poznan University of Medical Sciences, Poland
}

\begin{abstract}
Chemokines are important factors in the immunopathogenesis of multiple sclerosis. The objective of the study was to examine the CSF and serum levels of CXCL13 and CCL5 chemokines in primary progressive MS, compare results with relapsing remitting $\mathrm{MS}$ and control group with other noninflammatory neurological disorders. The levels of chemokines were measured by ELISA method. The CXCL13 CSF levels in PP and RR MS were higher in comparison with control group, without significant differences between these podgroups. Additionally CXCL13 level in PP MS inversely correlated with duration of the disease. CCL5 CSF level was also significantly higher in PP MS in comparison with control group. The results demonstrate involvement of CXCL13 and CCL5 chemokines in the immunopathogenetic mechanisms of primary progressive MS.
\end{abstract}

Keywords: chemokines, multiple sclerosis, immunopathology.

\section{Introduction}

About $10-15 \%$ of patients with multiple sclerosis represent primary progressive (PP) form with gradually increasing neurological disability. Patients with PP are older at onset as well as immunopathogenetic mechanisms show differences in comparison with relapsing remitting MS [1]. Although neuroaxonal degeneration underlines progressive disability in PPMS, inflammatory reactions are also present with evident microglial activation in healthy looking white matter additionally to cortical demyelination [2]. The extent to which inflammatory-immune reactions are involved in the pathogenesis of PP MS and its nature is not clear. Chemokines are important mediators in MS, in migration of inflammatory cells through blood brain barrier. The chemokine CXCL13 plays an important role in humoral immunity through recruitment of $B$ cells to the CNS and also T cell subsets expressing chemokine receptor CXCR5 [3]. RANTES (CCL5) mainly acts as a chemoattractant for activated $T$ cells and monocytes [4].

The objective of our study was to examine the CSF and serum levels of CXCL13 and CCL 5 chemokines in PP MS, compare the results with RR MS and control group with other noninflammatory neurological disorders.

Additional purpose was to evaluate correlation between CSF and serum levels of studied chemokines with age of patients, duration of the disease, IgG index and EDSS level.

\section{Material and Methods}

\section{Patients}

10 patients with PP MS (median age $42.3 \pm 9.6$ years, EDSS $3.5 \pm 1.2$ ), 15 patients with RR MS not on immunomodulatory treatment (median age $31.6 \pm 7.5$ years, 
Table 1. CXCL13 and CCL5 concentrations in patients with PP MS, RR MS and controls

\begin{tabular}{cccc}
\hline Mean \pm SD & PP MS & RR MS & Controls \\
\hline CXCL13 & & & \\
CSF $(\mathrm{pg} / \mathrm{ml})$ & $54.6 \pm 63.1$ & $44.6 \pm 48.6$ & $3.03 \pm 3.2$ \\
Serum $(\mathrm{pg} / \mathrm{ml})$ & $36.4 \pm 20.2$ & $55.1 \pm 35.6$ & $58.4 \pm 27.5$ \\
\hline CCL5 & & & \\
CSF $(\mathrm{pg} / \mathrm{ml})$ & $13.8 \pm 8.9$ & $7.9 \pm 2.9$ & $8.3 \pm 7.14$ \\
Serum $(\mathrm{ng} / \mathrm{ml})$ & $50.1 \pm 14.7$ & $48.4 \pm 19.0$ & $72.8 \pm 46.5$ \\
\hline
\end{tabular}

EDSS $1.8 \pm 0.9$ ) and 13 control patients (median age $36.2 \pm 7.4$ years) were included in the study. All patients with MS have fulfilled diagnostic criteria for RR and PP MS [5] control group included patients with noinnflammatory neurological disorders (tension headache $n=10$, vertigo $n=3$ ). Duration of the disease was $3.0 \pm 2.8$ years for PP MS group and $3.1 \pm 2.9$ years for RR MS group. The study was approved by the Ethics Committee of Poznan University School of Medicine. All patients involved in the study gave written informed consent.

CXCL13 and CCL5 measurements in the CSF and sera CSF and serum samples were frozen and stored at $-80^{\circ} \mathrm{C}$ until the analysis was performed. The levels of CXCL13 and CCL5 were measured by ELISA method according to the manufacturer's protocol (CXCL13/ BLC/BCA-1 Quantikine Human Colorimetric Sandwich ELISA, CCL5/RANTES Quantikine Human Colorimetric Sandwich ELISA (R\&D Systems). Plates were analyzed at OD $450 \mathrm{~nm}$ for $\mathrm{CXC13}$ and for CCL5 using Microplate Reader Elx 800 (Biotec Instruments Inc.). All assays were performed in duplicate. The intra- assay and inter- assay coeffifficients of variation were $2.5 \%$ and $10 \%$ respectively.

\section{Statistical analysis}

The nonparametric Mann-Whitney U test for comparison of two different groups and Spearmans' s rank test for correlations of the values were used for the statistical analysis. In all studied groups CSF and serum CXCL13 and CCL5 levels have been evaluated and correlated with $\lg \mathrm{G}$ index, age, duration of the disease and EDSS.

The work has been approved by the ethics committee and all human participants gave informed consent to the work

\section{Results}

In PP MS patients CSF CXCL13 level $54.6 \pm 63.1 \mathrm{pg} / \mathrm{ml}$ was significantly higher $(p<0.005)$ in comparison with control group $(3.03 \pm 3.2 \mathrm{pg} / \mathrm{ml})$. In RR MS patients CSF CXCL13 level $(44.6 \pm 48.6 \mathrm{pg} / \mathrm{ml})$ was also significantly higher in comparison with control group $(p<0.005)$. No significant differences in the CSF CXCL13 levels have been shown between PP and RR MS patients. CSF CXCL13 level in PP MS inversely correlated with duration of the disease, but not with age, IgG index or EDSS values.

In PP MS patients CSF CCL5 level $(13.8 \pm 8.9 \mathrm{pg} /$ $\mathrm{ml}$ ) was significantly higher $(\mathrm{p}<0.05)$ in comparison with control group $(8.3 \pm 7.14 \mathrm{pg} / \mathrm{ml}$.. No significant differences in the CSF CCL 5 levels have been observed between $\mathrm{PP}$ and RR patients.

No correlations of CSF CCL5 levels with age, duration of the disease, IgG index and EDSS values have been observed.

The serum CXCL13 and CCL5 levels did not differ significantly between studied groups and their values are presented in Table $\mathbf{1 .}$

\section{Discussion}

Our results demonstrate involvement of two important chemokines: CXCL13 and CCL5 in imunopathogenetic mechanisms in PP multiple sclerosis. CXCL13 chemokine is crucial for migration of $B$ cells [6]. The CXCL13 CSF level was higher in PP MS patients suggesting involvement of humoral immunity in this form of MS. Increased CSF CXCL13 chemokine levels were also observed in PP MS patients by Sellebjerg et al [3]. The CXCL13 CSF level inversely correlated with duration of the disease in PP MS patients, but not in RR MS, showing differences between these two forms of MS. This is the first such observation and should be confirmed on larger group of patients.

CXCL13 CSF level was also increased in RR MS patients. This confirms previous results by Krumbholtz et al [7] who has found also a significant correlation between CSF CXCL13 level in RR MS patients and number of $B$ cells and $T$ cells in the CSF.

The CCL5 chemokine (RANTES, regulated upon activation, normal $T$ cell expressed and secreted) is an agonist of CCR1, CCR3 and CCR5 receptors, which 
are expressed on target cells in MS plaques. We have not found increased CCL5 levels in the CSF of RR MS patients. Some previous studies revealed an increased CCL5 level in the serum and CSF of RR MS patients [4] while others failed to find any changes [8]. The inverse correlation between CSF CXCL13 levels and duration of the disease reflect decreasing immunological reactions with time course of the disease in PP MS patients.

\section{Acknowledgements}

\section{Conflict of interest statement}

The authors declare no conflict of interest.

\section{Funding sources}

There are no sources of funding to declare.

The authors thank Marlena Wójcicka and Elżbieta Kaufman for their technical support.

\section{References}

1. Iwanowski P, Losy J. Immunological differences between classical phenotypes of multiple sclerosis. J Neurol Scie. 2015;349:10-14.

2. Miller DH, Leary SM. Primary progressive multiple sclerosis. Lancet Neurol. 2007:6:903-912.

3. Sellebjerg F, Börnsen L, Khademi M, Krakauer MOT, Fredriksen JL, Sorensen PS. Increased cerebrospinal fluid concentrations of the chemokine CXCL13 in active MS. Neurology. 2009;73:2003-2010.

4. Sorensen TL, Tani M, Jensen J, Pierce V, Lucchinetti C, Folcik VA et al. Expression of specific chemokines and chemokine receptors in the central nervous system of multiple sclerosis patients. J Clin Invest. 1999;103:807815.
5. Polman Ch.H, Reingold SC, Banwell B, Clanet M, Cohen JA, Filippi et al. Diagnostic criteria for multiple sclerosis: 2010 revisions to McDonald criteria. Ann Neurol. 2011;69:292-302.

6. Gun MD, Ngo WN, Ansel KM, Ekland EH, Cyster JC, Williams LT. B-cell-homing chemokine made in lymphoid follicles activates Burkitt's lymphoma receptor-1. Nature. 1998;391:799-803.

7. Krumbholtz M, Theil D, Cepok S, Hemmer B, Kivisäkk $\mathrm{P}$, Ransohoff RM et al. Chemokines in multiple sclerosis: CXCCL12 and CXCL13 up-regulation is differently linked to CNS immune cell recruitment. Brain. 2006;129:200211.

8. Mahad DJ, Howell SJL, Woodroofe MN. Expression of chemokines in the CSF and correlation with clinical disease activity in patients with multiple sclerosis. J Neurol Neurosurg Psychiatry. 2002;72:498-502.

Acceptance for editing: 2016-12-10 Acceptance for publication: 2016-12-22

Correspondence address: Jacek Losy MD, PhD Department of Clinical Neuroimmunology Chair of Neurology Poznan Univeristy of Medical Sciences 49 Przybyszewskiego Street, 60-355 Poznan, Poland phone: +48 618691583 email: jlosy@amp.edu.pl 